\title{
Scale $r_{0}$ and the static potential from the CLS lattices
}

\section{F $L P H A$ \\ Collaboration}

$B U W-S C 2010 / 4$

WUB/10-37

\section{Björn Leder ${ }^{\star a, b}$, Francesco Knechtli ${ }^{a}$}

${ }^{a}$ Department of Physics, Bergische Universität Wuppertal

Gaussstr. 20, D-42119 Wuppertal, Germany

${ }^{b}$ Department of Mathematics, Bergische Universität Wuppertal

Gaussstr. 20, D-42119 Wuppertal, Germany

E-mail: leder@physik.uni-wuppertal.de

We report on the measurement of the static potential and the scale $r_{0}$ from HYP-smeared Wilson loops in two flavour QCD. We analyse the quark mass dependence of the potential and $r_{0}$ at three lattice spacings. We also compare the QCD static potential around distance $r_{0}$ with the static potential obtained from potential models.

The XXVIII International Symposium on Lattice Field Theory, Lattice2010

June 14-19, 2010

Villasimius, Italy

\footnotetext{
*Speaker.
} 


\section{Introduction}

The potential between two static colour charges in QCD is a quantity rich in features. At large distances $r$ between the two charges the phenomenon of string breaking is observed for the theory with dynamical quarks [1]. The quenched theory is expected to asymptotically coincide with bosonic effective string theory for $r \rightarrow \infty$ [2]. At intermediate distances a scale $r_{0}$ [3] can be defined which is readily computed in numerical simulations and can be related to phenomenological models of quarkonium. This scale can then be used to set the overall scale of a simulation and/or set the relative scale for simulations at different lattice spacings. Finally, at short distances the shape of the potential is described by renormalised perturbation theory. In fact, by taking the second derivative with respect to the distance a renormalised coupling can be defined. In this way the static potential connects the non-perturbative regime and the perturbative regime and is also a quantity where one expects large effects of dynamical quarks, i.e., large differences between the quenched and the unquenched result.

Here we report on an ongoing effort to measure the static potential on the configuration ensembles generated by CLS (Coordinated Lattice Simulations) ${ }^{1}$. They were generated with the DD-HMC software package ${ }^{2}$, which implements two degenerated flavours of improved Wilson fermions and the Wilson gauge action. There are ensembles at three different values of $\beta$ (i.e., three lattice spacings) and several values of the sea quark mass. The first objective is to provide the scale $r_{0}$. It can be compared to other methods of scale determination [4], used to compare dimensionless quantities among different collaborations, perform scaling analysis and preliminarily set the overall scale in physical units ${ }^{3}$. Since the data presented here are not based on all the available statistics and some details of the analysis might change, the results presented here are preliminary.

The report is organised as follows. In the next section the techniques used to extract the static potential with low statistical fluctuations and small systematic errors are summarised (see [5] for more details). In section 3 the scale $r_{0}$ is determined and extrapolated to vanishing sea quark mass. In section 4 applications beyond scale setting are collected, i.e., a comparison of the quark mass dependence of $r_{0}$ with other collaborations and the determination of a renormalised coupling.

\section{The static potential}

To determine the static potential $V(r)$ we measure rectangular Wilson loops. They have extension $t$ and $r$ in temporal and spatial direction respectively. Schematically, we measure

$$
C(t)=\langle t \underset{r}{\stackrel{r}{\square}} t\rangle, \quad r \text { fixed, }
$$

where the brackets denote the expectation value with respect to the two flavour QCD measure and the square represents the product of link variables around a closed rectangular path. This is equivalent to a static quark-anti-quark pair at spatial separation $r$ propagating distance $t$ in time. For large euclidean time separations the signal is dominated by the ground state, which coincides with the static potential

$$
C(t) \stackrel{t \rightarrow \infty}{=} A(r) \mathrm{e}^{-V(r) t}, \quad r \text { fixed } .
$$

\footnotetext{
${ }^{1}$ https://twiki.cern.ch/twiki/bin/view/CLS/WebHome

${ }^{2}$ http://luscher.web.cern.ch/luscher/DD-HMC

${ }^{3}$ Because of the uncertainty coming from the phenomenological models used to give $r_{0}$ in physical units, this is somewhat unsatisfying and should be seen as an intermediate step.
} 


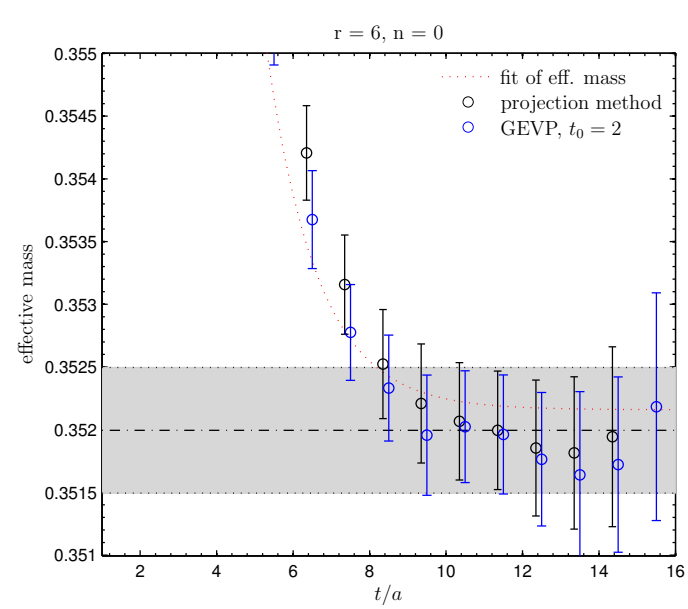

Figure 1: Effective "mass" plot for the Wilson loop correlator matrix for $r=6 a$ and $t_{0}=2 a$. See text for a detailed explanation.

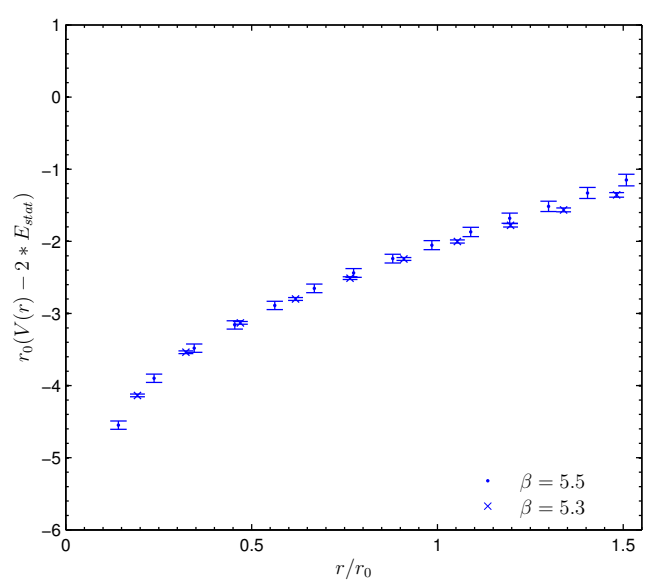

Figure 2: Static potential at two values of the lattice spacing. The static energy $E_{\text {stat }}[8]$ is subtracted to obtain a renormalised quantity.

A straightforward determination of $V(r)$ from this definition with the original link variables of the configurations is bound to suffer from large statistical and systematic errors for two reasons. First, the signal to noise ratio will decrease exponentially for large $r$ and $t$ due to ultraviolet fluctuations. Second, the overlap with the ground state (encoded in $A(r)$ in eq. (2.2)) will be poor.

To remove the ultraviolet fluctuations the original link variables are replaced by smeared links. To this end one level of hypercubic smearing [6] with parameters $\alpha_{1}=1.0, \alpha_{2}=1.0, \alpha_{3}=0.5$, referred to as HYP2 [7] was found to give the best result. The whole analyses was carried out with a second parameter set $\alpha_{1}=0.75, \alpha_{2}=0.6, \alpha_{3}=0.3$, referred to as HYP1. When not specified, the parameter set HYP2 is used. The smearing of the temporal links can be understood in terms of choosing an action for the static quarks and the smearing of the spatial links in terms of a redefinition of the operator that creates the static quark-anti-quark pair (see [5] for a detailed derivation). Using smeared links greatly improves the signal to noise ratio at large time separations.

Since the exact wave function of the desired ground state is unknown the usage of a variational method is mandatory to improve the overlap. As already explained further smearing the spatial links on the left hand side of eq. (2.1) is equivalent to a redefinition of the operator that creates the static quark-anti-quark pair. Intuitively, by smearing the generated sate becomes more and more extended. This way one obtains a correlator matrix

$$
C_{l m}(t)=\langle t \underset{r, l}{\stackrel{r, m}{\square} t}\rangle, \quad r \text { fixed } .
$$

In particular the indices $l, m=1, \ldots, M$ specify the $n_{l, m}$ levels of spatial HYP smearing with parameters $\alpha_{2}=0.6, \alpha_{3}=0.3^{4}$ that are applied to the spatial links. Throughout this report we use $M=4$ and $n_{l}=l-1$.

From the correlator matrices (2.3) effective "masses" are extracted with two different methods. The starting point in both cases is the generalised eigenvalue problem (GEVP)

$$
C(t) \psi_{\alpha}\left(t, t_{0}\right)=\lambda_{\alpha}\left(t, t_{0}\right) C\left(t_{0}\right) \psi_{\alpha}\left(t, t_{0}\right), \quad \alpha=0, \ldots, M-1 .
$$

\footnotetext{
${ }^{4}$ Only spatial links are involved, thus only two parameters are needed.
} 


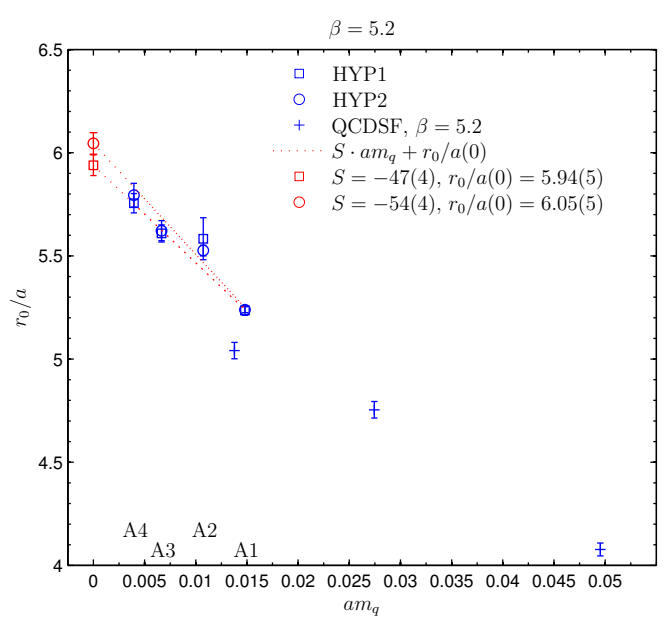

Figure 3: Chiral extrapolation of $r_{0} / a$ at $\beta=5.2$. QCDSF data from [12] for comparison.

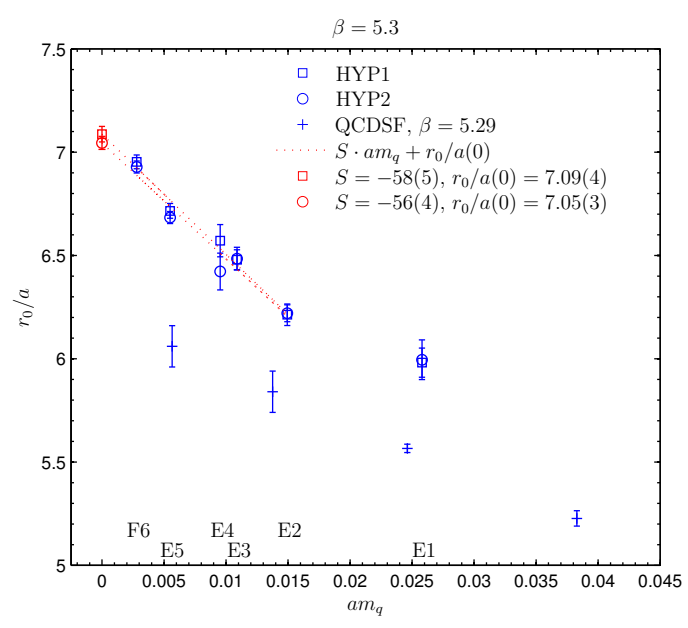

Figure 4: Chiral extrapolation of $r_{0} / a$ at $\beta=5.3$. QCDSF data from [12] for comparison.

From the generalised eigenvalues one directly obtains

$$
E_{\alpha}\left(t+\frac{a}{2}, t_{0}\right) \equiv \ln \left(\lambda_{\alpha}\left(t, t_{0}\right) / \lambda_{\alpha}\left(t+a, t_{0}\right)\right)=E_{\alpha}+\beta_{\alpha} \mathrm{e}^{-\left(E_{M}-E_{\alpha}\right)\left(t+\frac{a}{2}\right)}+\ldots
$$

where $E_{0} \equiv V(r)$ and the higher states contributions are expected to die out with large $E_{M}-E_{\alpha}$ or faster. In [9] the authors were able to prove this for $t \leq 2 t_{0}$. In fig. $1 E_{0}\left(t+\frac{a}{2}, t_{0}\right)$ is plotted for an intermediate $r$ and $t_{0}=2 a$ (blue circles) ${ }^{5}$. In order to quantify the contribution of higher states we perform a fit with the two terms of the right hand side of eq. (2.5) including data points for $\alpha=0,1$ and $t \leq 2 t_{0}$ (red dotted curve). The term modelling the higher states is used to estimate the systematic error due to them when extracting the ground state at a given time $t$ (see below).

Finally, the projection method of [11] is used to obtain a second determination of $E_{\alpha}(t+$ $\left.\frac{a}{2}, t_{0}\right)$. There the correlator matrices are projected to the ground state generalised eigenvector $v=\psi_{0}\left(t_{0}+a, t_{0}\right)$

$$
f(t)=v^{T} C(t) v
$$

The resulting numbers are locally fitted by a single exponential, i.e. three successive values $f\left(t_{p}\right)$, $t_{p}=t-a, t, t+a$ are fitted to $f\left(t_{p}\right)=b \mathrm{e}^{-E_{0}\left(t, t_{0}\right) t_{p}}$ (black circles in fig. 1).

With the parameters as in this report we found the projection method to be slightly more stable, i.e. to exhibit longer plateaus. Therefore we extract the static potential from the black circle in fig. 1 with the smallest sum of statistical and systematic error (black dashed-dotted curve and grey error band).

Applying this procedure to all values of $r$ we obtain the static potential as a function of $r$. In fig. 2 we plot the result (made dimensionless by multiplying with $r_{0}$, see below) for two values of the lattice spacing at roughly the same sea quarks mass. The plot shows that lattice artefacts are small.

\footnotetext{
${ }^{5}$ Statistical errors are determined using the method and program of [10].
} 


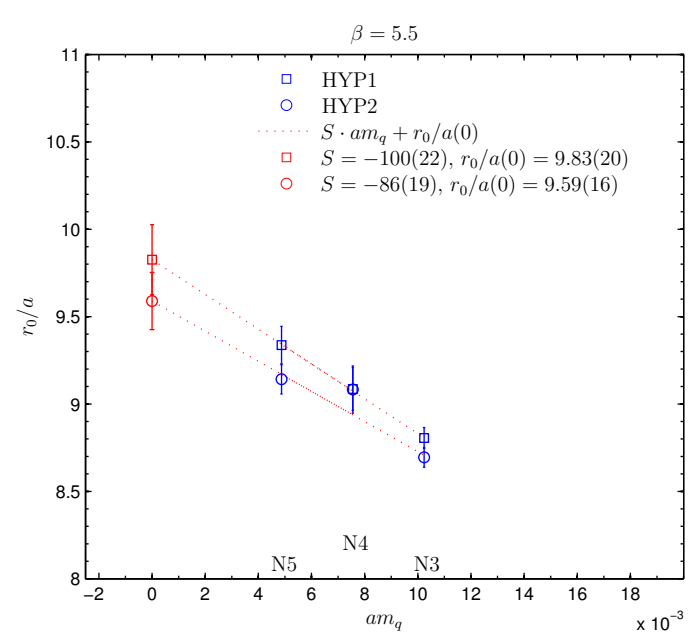

Figure 5: Chiral extrapolation of $r_{0} / a$ at $\beta=5.5$.

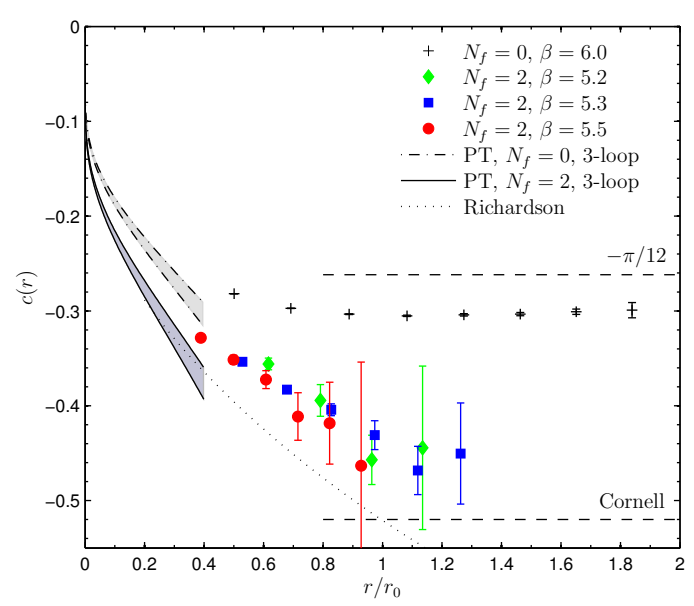

Figure 6: Renormalised quantity $c(r) . N_{f}=2$ data are at $r_{0} M_{P S} \approx 1$. See text for details.

\section{Scale $r_{0}$}

The scale $r_{0}$, introduced in [3], is defined in terms of the static force $F(r)=V^{\prime}(r)$ by solving

$$
\left.r^{2} F(r)\right|_{r=r_{0}}=1.65 .
$$

Its physical value is $r_{0} \approx 0.5 \mathrm{fm}$, thus it is sensitive to the non-perturbative character of the theory. Off the lattice it can only be determined through phenomenological potential models. On the lattice the static force is computed from the potential as the finite difference

$$
F\left(r_{I}\right)=\frac{1}{a}[V(r)-V(r-a)]
$$

where $r_{I}=r-a / 2+\mathrm{O}\left(a^{2}\right)$ is chosen such that in (3.2) at tree level all lattice artefacts cancel out. To solve (3.1) the force is then locally parametrised by $a^{2} F(r)=f_{0}+f_{2} a^{2} / r^{2}$. Taking the two values that enclose the solution, the parametrisation is uniquely determined as well as $r_{0} / a$. This lattice definition of $r_{0} / a$ ensures that it is a smooth function of the bare parameters $\beta$ and $m_{0}$. To estimate the systematic uncertainties due to the parametrisation the same procedure is repeated with an additional term $f_{4} a^{4} / r^{4}$ and three successive $r$-values. They are found to be negligible.

We analysed three run sets at three different lattice spacings with $\beta=5.2,5.3,5.5$. At each lattice spacing there are runs at 3-6 quark masses. For a scale setting we decided to extrapolate to the chiral point. In figs. 3-5 the measured values of $r_{0} / a$ are plotted versus the subtracted quark mass $a m_{q}=a m_{0}-a m_{\mathrm{cr}}$. Here we give the result for both parameter sets HYP1 and HYP2. The extrapolation to $a m_{q}=0$ is done via a linear fit. The result of the fits are given in the legends of the plots. The HYP2 results are compatible with the HYP1 ones and have the smaller error. Thus we list here for $r_{0} / a(\beta)$ in the chiral limit:

$$
r_{0} / a(5.2)=6.05(5), \quad r_{0} / a(5.3)=7.05(3), \quad r_{0} / a(5.5)=9.59(16)
$$

Using our new determination of $r_{0} / a$ we get a preliminary update on the $\Lambda$-parameter of [7]:

$$
r_{0} \Lambda_{\overline{\mathrm{MS}}}^{N f=2}=0.73(3)(5),
$$



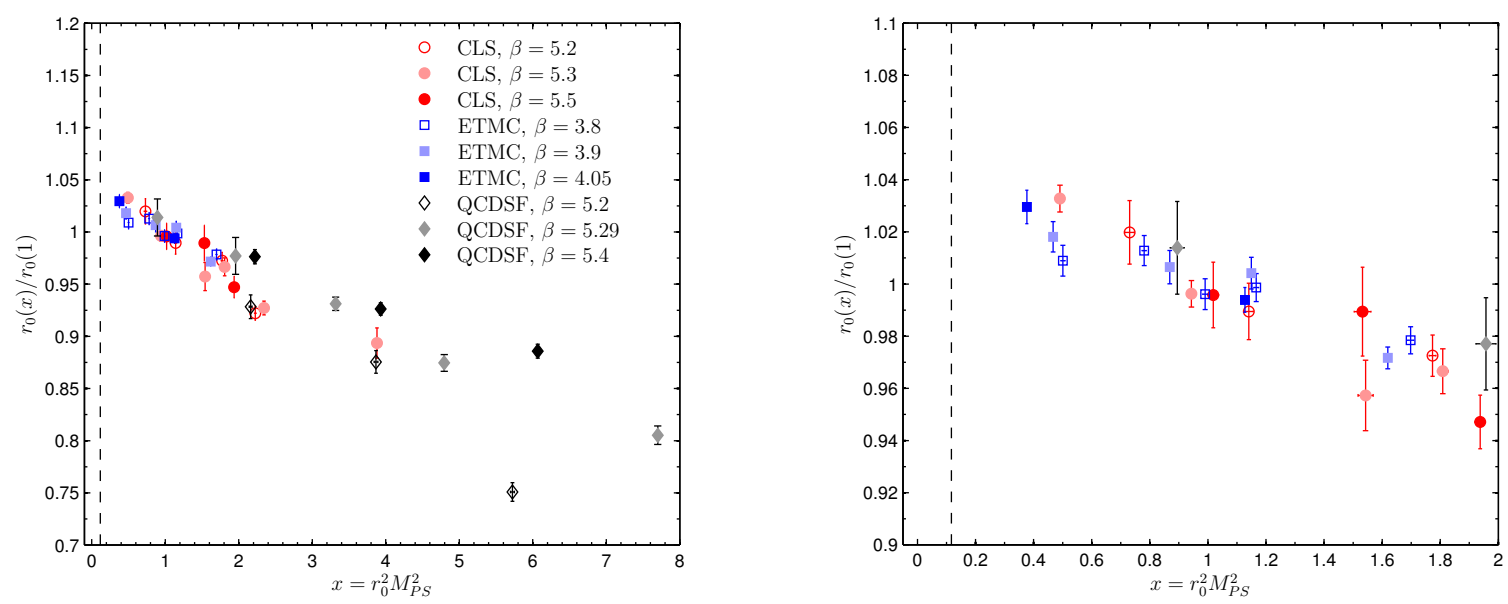

Figure 7: Plot of dimensionless and physical quantities comparing different efforts to simulate two flavour QCD. The right panel is a blow-up of the region $x=0 \ldots 2$.

where the first error comes from $r_{0} / a$ and the second from the running of the coupling.

In figs. 3 and 4 we also plot data from the QCDSF collaboration [12]. Since they are using exactly the same action, the results at coinciding bare parameters should agree within errors. The most tension is observed between their lightest point and the heaviest of this report at $\beta=5.2$ in fig. 3. Also in fig. 4 the difference is hardly explainable by the difference in the $\beta$-values (5.3 versus 5.29). The most plausible explanation is that they used a global fit including many terms to model the potential (and thus the force) ${ }^{6}$ over a wide range of $r$ values. This way the statistical error in $r_{0} / a$ is reduced, but at the price of large systematic uncertainties.

\section{Physics results}

First, we present a quantity for which large effects of dynamical quarks can be observed. A renormalised, physical quantity can be defined in terms of the derivative of the static force

$$
c(r)=\frac{1}{2} r^{3} F^{\prime}(r) .
$$

In [2] it was determined with high precision in pure gauge theory. With the data presented here we are able to compare the pure gauge case to the $N_{f}=2$ theory. On the lattice we write $c$ in terms of a finite difference

$$
c(\tilde{r})=\frac{1}{2 a^{2}} \tilde{r}^{3}[V(r+a)+V(r-a)-2 V(r)],
$$

where $\tilde{r}=r+\mathrm{O}\left(a^{2}\right)$ is chosen such that at tree level all lattice artefacts cancel out. In fig. 6 we plot $c$ for the three values of $\beta$ at roughly the same sea quark mass $\left(r_{0} M_{P S} \approx 1\right)$. For comparison we also plot the $N_{f}=0$ data [2]. The analytic curves in the plot are the 3-loop perturbative curves (dashed-dotted for $N_{f}=0$ and solid line for $N_{f}=2$, spread due to uncertainty in the $\Lambda$-parameter) ${ }^{7}$, the universal value $-\pi / 12$ from bosonic string theory $[13,14]$ (that the $N_{f}=0$ data is approaching asymptotically for $r \rightarrow \infty$ ), the value of $c$ in the Cornell [15] potential and the curve derived from the Richardson [16] potential.

\footnotetext{
${ }^{6}$ private communication with P. Rakow

${ }^{7}$ For details on the perturbative expressions for $c(r)$ and how it is related to a renormalised coupling we refer the reader to [5].
} 
Second, we present in fig. 7 a comparison to other efforts simulating QCD with two light quarks. For the x-axis we define a dimensionless quantity $x=r_{0}^{2} M_{P S}^{2}$ (where $M_{P S}$ is the pseudoscalar mass). For the y-axis we define the ratio of $r_{0}(x) / r_{0}(1)$, where $x=1$ serves as a reference point to cancel the unknown overall scale. We have chosen to include QCDSF [12] and ETMC [17], because of the readily available data for $r_{0}$ and $M_{P S}$. The left panel shows the whole range of data points, whereas the right panel is a blow-up of the region close to the physical point. Assuming a physical value $r_{0}=0.5 \mathrm{fm}$ the physical value of $x=r_{0}^{2} M_{P S}^{2}$ is indicated by the vertical dashed line. As one can see the spread of the points is narrowing towards the physical point, where they start to agree within errors.

\section{Acknowledgements}

We thank Rainer Sommer for helpful discussions on various aspects of this work, Nazario Tantalo for extensive checks of the Wilson loop measurements, Stefan Schäfer for help in checking the HYP smearing and providing some $a m_{\mathrm{cr}}$ values, and Nikos Ingres for discussions on the quantity $c(r)$. We further thank the Forschungszentrum Jülich and the Zuse Institut Berlin for allocating computing resources to this project. Part of the Wilson loop measurements were performed on the PAX cluster at DESY, Zeuthen.

\section{References}

[1] SESAM, G.S. Bali, H. Neff, T. Duessel, T. Lippert and K. Schilling, Phys. Rev. D71 (2005) 114513, [hep-lat/0505012].

[2] M. Lüscher and P. Weisz, JHEP 0207 (2002) 049, [hep-lat/0207003].

[3] R. Sommer, Nucl.Phys. B411 (1994) 839, [hep-lat/9310022].

[4] B. Brandt et al., (2010), [arXiv:1010.2390], PoS (Lattice 2010 ) 164.

[5] M. Donnellan, F. Knechtli, B. Leder and R. Sommer, to appear soon.

[6] A. Hasenfratz and F. Knechtli, Phys.Rev. D64 (2001) 034504, [hep-lat/0103029].

[7] ALPHA Collaboration, M. Della Morte et al., Phys.Lett. B581 (2004) 93, [hep-lat/0307021].

[8] B. Blossier et al., (2010), PoS (Lattice 2010) 308.

[9] B. Blossier, M. Della Morte, G. von Hippel, T. Mendes and R. Sommer, JHEP 04 (2009) 094, [arXiv:0902.1265].

[10] ALPHA, U. Wolff, Comput. Phys. Commun. 156 (2004) 143, [hep-lat/0306017].

[11] ALPHA collaboration, M. Guagnelli, R. Sommer and H. Wittig, Nucl.Phys. B535 (1998) 389, [hep-lat/9806005].

[12] QCDSF/UKQCD Collaboration, D. Brommel et al., Eur.Phys.J. C51 (2007) 335, [hep-lat/0608021].

[13] M. Lüscher, K. Symanzik and P. Weisz, Nucl. Phys. B173 (1980) 365.

[14] M. Lüscher, Nucl. Phys. B180 (1981) 317.

[15] E. Eichten, K. Gottfried, T. Kinoshita, K.D. Lane and T.M. Yan, Phys. Rev. D21 (1980) 203.

[16] J.L. Richardson, Phys. Lett. B82 (1979) 272.

[17] ETM Collaboration, R. Baron et al., JHEP 1008 (2010) 097, [arXiv:0911.5061]. 